Journal of Applied Fluid Mechanics, Vol. 1, No. 1, pp. 71-77, 2008. Available online at www.jafmonline.net, ISSN 1735-3645.

DOI: $10.36884 / \mathrm{jafm} .1 .01 .11839$

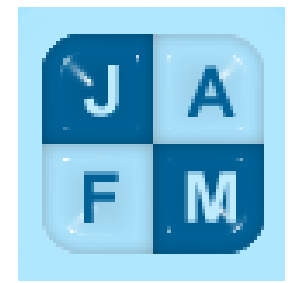

\title{
Turbulent Separated Flows: Near-Wall Behavior and Heat and Mass Transfer
}

\author{
A. Gorin \\ School of Engineering and Science, Curtin University of Technology, Sarawak Campus, Miri, Sarawak, CDT \\ 250, 98009, Malaysia \\ Email: alexander.g@curtin.edu.my
}

(Received February 25, 2007; accepted May 19, 2007)

\begin{abstract}
This paper was presented at the Eleventh Asian Congress of Fluid Mechanics as an invited/keynote lecture. The lecture summarizes development of physical model for transfer processes in turbulent separated flows. Attention is focused on the factors that have led to significant advances in understanding of the mechanisms of transfer processes in separated flows, which differs from those in traditional attached turbulent flows in channels and zero pressure gradient turbulent flows. The physical model of transfer processes in the near-wall region of turbulent separated and reattached flows based on the assumption of the governing role of generated local pressure gradient that takes place in the immediate vicinity of the wall in separated flow as a result of intense instantaneous accelerations induced by large-scale vortex flow structures is discussed. Similarity laws for mean velocity and temperature profiles and spectral characteristics of the transfer processes resulting in the physical model are confirmed by the available experimental data. The physical model has provided explanations of the well-known empirical heat and mass transfer correlations for turbulent separated flows and other types of flows close in their structure to those of turbulent separated flows.
\end{abstract}

Keywords: structure of turbulent separated flows, transport mechanisms, velocity profile, temperature profile, velocity fluctuation spectra, similarity laws, 'two-thirds power law'.

\section{INTRODUCTION}

Turbulent separated flows (TSF) occur commonly at sufficiently high Reynolds numbers in many technological applications of great importance to mechanical, aerospace, chemical, and civil engineers. Such flows are observed in flows of interest to meteorologists and earth scientists.

By turbulent separated flow, we mean the entire process of the turbulent boundary layer separation and reattachment onto a solid surface. It may be generated by strong adverse pressure gradients in or on smoothly varying geometries (the first type of TSF): flow in a diffuser, on airfoils, or over turbine blades; and flows that separate due to abrupt changes in geometry with geometrically fixed location of separation. Flows in cavities and over a single-sided backward-facing step, flows downstream of the leading edge of a blunt plate or a blunt circular cylinder, flow behind a normal plate with a long splitter plate are examples of the second type.

The occurrence of flow separation and reattachment affects fundamental transport characteristics and results in severe change in the performance of fluid machinery and heat and mass transfer devices.

Separation of turbulent boundary layer is reported in experimental studies to be unsteady phenomenon consisting of local events of unsteady separations which occurs chaotically in the vicinity of the mean flow separation line. Each such separation is accompanied by departure of shear layer which in its turn generates new large eddies, with decreasing the average frequency due to rapid growth and agglomeration with one another. They supply the turbulence energy to the near wall flow.

Experimental studies of turbulent flows over sharp-edged bluff bodies demonstrate the occurrence of the persistent large-scale eddies that are developed in the separated freeshear line. The instantaneous reattachment (the impingement of the separated shear layer on the wall) location fluctuates with frequency which corresponds to that of wall pressure fluctuations with maximum energy content. Furthermore, there is a very low frequency vertical or flapping motion of the reattaching shear layer which causes the turbulence-intensity level of the detached flow to increase by $5-10 \%$ in comparison with the planemixing layers with negligible contributions to the Reynolds shearing stress. 
Structurally, a separation-and-reattaching region of flow is considered as containing a shear layer that on one side bounds a main stream and on the other, next to a wall, there is a region of instantaneous or mean, relatively slow reverse flow with a thin near-wall zone. Namely the flow pattern and structural characteristics of the near-wall zone are responsible for heat and mass transfer in separated flows. The thickness of the near-wall zone corresponds to the distance from the wall to the peak mean reverse flow. One can expect that the flow structure of this zone is very different from an attached turbulent boundary layer, and that is the case. Some the most characteristic properties of the near-wall zone of turbulent separated flows and regularities of heat and mass transfer as it has been shown in experimental studies (a quite extensive list of references one can find in reviews by Eaton and Johnston (1983); Simpson (1989); Chang (1970) on this class of flows) are as follows:

- breakdown of the logarithmic law-of-the-wall, so that outside of the linear sublayer the friction velocity is not the characteristic scale for mean velocity generalization;

- profiles of turbulence intensity in the near-wall region do not have a peak close to a wall;

- the Reynolds shear stress remains small in the near-wall region;

- the intensity of velocity fluctuations parallel to the wall in the near-wall region is of the order of magnitude of the local mean velocity.

- the intensity of fluctuations normal to the wall is nearly an order of magnitude smaller.

- the Nusselt number is usually proportional to the $2 / 3$ power of the Reynolds number in contrast to attached turbulent boundary layers with the exponent close to 0.8 .

- the Nusselt number reaches its maximum value at the point where the mean skin friction passes through zero that show the Reynolds analogy fails in this type of flow.

Theoretical description of complex turbulent flows is based on four main approaches: Reynolds averaged Navier-Stokes (RANS) approach; large eddy simulation (LES); direct numerical simulation (DNS); hybrid approaches (an extensive list of references can be found in reviews by Launder (1988), Pletcher (1988), Speciale (1991), Churchill (2000), and Hanjalic (2005)).

Among conventional turbulence modeling methods, as representatives of RANS approach, the k- models have become most popular and applicable, in conjunction with wall damping functions, to many practical complex flows owing to their relative simplicity and reasonable computing time. But such wall functions are not universal, and the constants of damping functions need to be adjusted for different Reynolds numbers and flows. A number of extended versions of the $\mathrm{k}$ - models were proposed to deal with the turbulence anisotropy.

For predicting the anisotropy of Reynolds stresses, Reynolds stress models are used as well. But in this case higher-order correlations in the highly nonlinear coupled second moment differential transport equations derived in exact form need to be modeled with assumptions of questionable validity especially in the near-wall zone.
Advent of supercomputers has made possible, in principle, direct numerical simulation (DNS) of turbulent flows in which the full unsimplified and unaveraged, threedimensional and time-dependent transport equations are solved directly without the use of turbulent models. The main limitation is that the grid spacing is required to be reduced with an increase in the flow rate.

Large eddy simulation predicts explicitly the large eddy dynamics, their three-dimensional and unsteady motion. The smaller eddies are assumed to be isotropic and universal, and the $\mathrm{k}$ - model or its equivalent is used for their simulation, of course with the shortcomings intrinsic to the RANS approach. The grid-size must be fine enough to allow the resolution of the dissipation scale length in a large enough computational domain related to the largest turbulence scale or the characteristic size of physical device.

Even with great prospects for simulation of complex turbulent flows with DNS and LES, the direct numerical simulation owing to computing time and memories problems is an useful tool to improve our physical insight into turbulence mechanism than to predict engineering parameters, whereas LES proved to be closer to engineering design problems is limited to relatively low Re numbers and simple geometry. In view of formidable limitations and constrains of each strategy by itself, in recent years real impetus has been given to hybrid approaches representing a compromise between different strategies. One such example is a combination between the potential of LES and relative simplicity and reasonable computational economy and accuracy of RANS in application to high Reynolds number flows and heat transfer in engineering practice.

The foregoing shows that although turbulent separated flows are frequently observed, and despite of the extensive experimental studies and advances in numerical simulation the prediction of fluid dynamics and heat and mass transfer in such flows is still the challenging problem for the modern turbulence theory and computational fluid dynamics. The laws that govern momentum, heat and mass transfer in turbulent separated flows exhibit characteristic features which are poorly understood, there are no theoretical concepts to generalize experimental data, to test the consistency of experimental and computed values (of numerical predictions). To provide asymptotic solutions, physically justified turbulence model taking into consideration physical mechanism and main governing parameters should be developed.

The objective of this lecture is to present such a theoretical model based on fundamental transport mechanisms and to discuss its perspectives and backlog of experience for developing limiting laws, regularities and recommendations on construction of correlating equations for heat and mass transfer in turbulent separated flows. 


\section{SIMILARITY LAWS AND HEAT TRANSFER}

Main properties of the flow in the near-wall zone of turbulent separated flows have been discussed in the introductory part. Some quantitative characteristics need to be represented as a base for a physical model of transfer processes in the near-wall zone.

Our estimations (see, for example, Gorin and Sikovsky, 1995) of the scales of turbulent motion in the near-wall zone of separated flow on a flat plate (data by Simpson et al., 1981) show that the wavelength $\lambda$ of turbulent disturbances has the order of $\lambda / N \propto 33$, where $N(0<y<N)$ usually called as the near-wall zone of recirculation flow, is a location of a maximum backflow velocity (Fig.1).

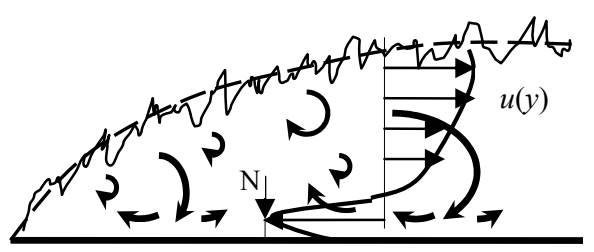

Fig. 1- Turbulent separated boundary layer

The backflow velocity has a maximum at a distance $N$ from the wall that is far smaller than the local boundary layer thickness - in experiments by Simpson et al. (1981) it constituted a few percent of the local boundary layer thickness.

Thus, the spatial dimensions of flow-frequency fluctuations of the longitudinal velocity in the near-wall zone are much greater than the layer thickness. On the other hand, according to the measurements by Simpson (1989), the rootmean-square fluctuation of the transverse velocity in the near-wall zone is an order of magnitude lower than the longitudinal fluctuations (we included this result among the most characteristic features of the near-wall zone of turbulent separated flows in the introductive part of the lecture). These facts indicate that the large-scale motion in the near-wall zone is of the character of an unsteady boundary layer. In this case large-scale unsteady recirculation flow supported by large-scale vortices from the mixing layer above plays the role of external flow. Since the fluctuation level is of the order of the mean velocity, unsteady motion in the boundary layer is characterized by large amplitude.

Thus turbulent motion in the boundary layer can be divided into two components: a large-scale component with a length scale much greater than the near-wall thickness $N$ and a small-scale component which is composed of vortices with a size of the order of $N$ and smaller.

Averaging the Navier-Stokes equations with the Reynolds averaging rules over small-scale fluctuations and assuming no correlations between large-scale and small-scale velocity fluctuations, one can obtain the equations of large-scale motion in the near-wall layer (Gorin and Sikovsky, 1995,
1996, 1997):

$\frac{\partial \vec{u}}{\partial t}+(\vec{u} \nabla) \vec{u}+v \frac{\partial \vec{u}}{d y}+\alpha=\frac{\partial \vec{\tau}}{\partial t}+v \frac{\partial \vec{u}}{\partial y^{2}}$

$\nabla \cdot \vec{u}+\frac{\partial v}{\partial y}=0$

where $\vec{u}=(u, w)$ is the large-scale velocity vector, $u, w$ are the $x$ - and $z$-components of $\vec{u}, \nabla=(\partial / \partial x, \partial / \partial z)$, $\vec{\alpha}=\nabla p$ is the kinematical large-scale pressure gradient, $\vec{\tau}=-\left(\left\langle u^{\prime} v^{\prime}\right\rangle,\left\langle w^{\prime} v^{\prime}\right\rangle\right)$ are the turbulent stresses created by the small-scale fluctuations denoted by primes, angle brackets denote an average over the small scales. The pressure gradient $\alpha^{\prime}$, constant across boundary layer, is created by the large-scale outer motions.

For distances $\mathrm{y}<<N$ convective terms could be omitted so that from $\mathrm{Eq}(1)$ it follows

$$
\vec{\tau}=\vec{\tau}_{w}+\vec{\alpha} y-v(\partial \vec{u} / \partial y)
$$

This relationship explains choice of the wall shear stress $\tau_{w}$ and $\alpha$ as governing parameters for the inner region of the near-wall layer outside of the viscous sublayer.

The outlined considerations could be represented as the first hypothesis:

Hypothesis 1. When the Reynolds number is high enough, the statistical regimes of large- and small-scale velocity fluctuations are independent. Statistical regime of smallscale velocity fluctuations is determined uniquely in the near-wall zone $y<<N$ by the local values $\vec{\tau}_{w}(\vec{x}, t), \vec{\alpha}(\vec{x}, t)$ and $v$.

One can show (Gorin and Sikovsky, 1996) that for the critical value of the dimensionless criterion formed from the parameters involved in Eq (3), $P_{+}=v \alpha / \tau_{w}^{3 / 2} \sim 3 \cdot 10^{-3}$ (physically, the criterion $P_{+}$represents the ratio of the characteristic viscous length, $\delta=v / \tau_{w}^{1 / 2}$, and $\delta_{p}=\tau_{w} / \alpha$, characterizing relative contribution of the wall shear stress and large-scale pressure gradient) the constant-stress sublayer degenerates. In other words, there is no the logarithmic wall law! When $P_{+}<<10^{-2}$ the second term in Eq (3) can be omitted, and ordinary zero-pressuregradient turbulent boundary layer is realized with the friction velocity as a velocity scale. In terms of the wall law coordinates, if $P_{+} \rightarrow 0$ within the region $1<<y_{+}<<P_{+}$ the effect of the pressure gradient is negligibly small $\left(y_{+}=\tau_{w}^{1 / 2} y / v\right)$.

If $P_{+}=v \alpha / \tau_{w}^{3 / 2}>>3 \cdot 10^{-2}$, and this is the case for the backflow region, the wall shear stresses become negligible that corresponds to the flow near the separation; fluid acceleration in the near-wall layer that is produced by 
large-scale vortices of separated flows prevents formation of a sublayer of constant shear stress. In this connection, the friction velocity is not the governing parameter of the nearwall layer of turbulent separated flows. The statistical regime of turbulent flow in this region is determined by the local pressure gradient (local instantaneous accelerations) and distance from surface. The root-mean-square wall pressure gradient is a quantitative characteristic of these accelerations.

Thus we may put forward the following similarity hypothesis:

Hypothesis 2. When $P_{+}$is high enough $\left(P_{+}>>10^{-2}\right)$ the statistical regime of the small-scale fluctuations in the nearwall region $y<<N$ is determined uniquely by the values $v$ and $\vec{\alpha}(\vec{x}, t)$.

From this hypothesis and the dimensionality considerations, it follows that the thickness of the viscous sublayer is given by

$l \propto v^{2 / 3} \alpha^{-1 / 3}$

And for distances from the wall far greater than the viscous layer thickness it would appear reasonable to formulate the third hypothesis:

Hypothesis 3. If the conditions of the first and second hypotheses are complied with, there is a range of distance from the wall $v^{2 / 3} \alpha^{-1 / 3}<<y<<N$ for which statistical regime of the small-scale fluctuations in the near-wall region is determined uniquely by the value $\vec{\alpha}(\vec{x}, t)$ and does not depend on viscosity $v$.

These hypotheses summarize physical model of transfer processes in the near-wall layer of turbulent separated flows and serves as a base for theoretical analysis.

From dimensional considerations (Gorin and Sikovsky, 1995, 1996, 1997), similarity laws for the near-wall mean velocity and temperature, and small-scale spectra of longitudinal velocity and pressure fluctuations were obtained (and which are very different from those for isotropic turbulence - Kolmogorov's similarity laws):

$$
\begin{aligned}
& \vec{u}=\frac{\vec{\alpha}}{\alpha}\left[K(\alpha y)^{1 / 2}+K_{1}(v \alpha)^{1 / 2}\right], \\
& T=K_{t} \frac{q}{\rho c(\alpha y)^{1 / 2}}+K_{t 1} \frac{q}{\rho c(v \alpha)^{1 / 3}}, \\
& E_{i j} \propto \alpha_{r m s} k_{x}^{-2}, E_{P} \propto \rho^{2} \alpha_{r m s}^{2} k_{x}^{-3}
\end{aligned}
$$

where $K, K_{1}$ are constants and $K_{t}$ and $K_{t 1}$ are the universal functions of Prandtl number $\operatorname{Pr}=v / a, a$ is the thermal diffusivity, $q$ is the wall heat flux, $\alpha_{r m s}$ is a root-meansquare wall pressure gradient, $k$ is a longitudinal wavenumber.
Heat transfer within shear stress layer can be treated with the similar arguments with the large-scale heat transfer equation reduced to $q=-\rho c_{0}\left\langle v^{\prime} T^{\prime}\right\rangle$.

The heat transfer law after averaging over the ensemble of large-scale fluctuations of wall pressure gradient is obtained in the form

$$
\mathrm{Nu}=\operatorname{const} C_{\alpha}^{1 / 3} \operatorname{Re}^{2 / 3} \operatorname{Pr}^{\beta}
$$

Since the boundary layer accelerations due to large-scale (of the scale $L_{V}$ ) vortices $\alpha_{r m s} \propto U_{\infty}^{2} / L_{V} \quad$ (it is worth noting that $\alpha_{r m s}$ is $\alpha_{r m s}^{2}=[(1 / \rho)(d P / d x)]^{2}+\alpha_{r m s}^{\prime 2}$, where $\alpha_{r m s}^{\prime 2}$ is the near-wall root-mean-square pressure gradient fluctuation), coefficient $C_{\alpha}=\alpha_{r m s} L_{V} / U_{\infty}^{2}$ is independent of the Reynolds number and law (5) represents the " $2 / 3$ power law" $\left(\mathrm{Nu} \propto \mathrm{Re}^{2 / 3}\right)$ for turbulent heat transfer in separated flows.

Thus, the "2/3 power law", which was found experimentally in early 1960s (see the survey, Chang, 1970) and is encountered in almost all heat transfer experiment with turbulent separation and similarity laws for structural characteristics of the near-wall region of turbulent separated flows have received theoretical justification for the first time.

\section{DISCUSSION OF SOME RESULTS}

Figure 2 represents longitudinal velocity fluctuation spectra $F(k)=E_{x x}(k) / u^{\prime 2}$ measured near the bottom of the square (a) and rectangular (b) cavities in the middle section (Varfolomeev et al., 1984).

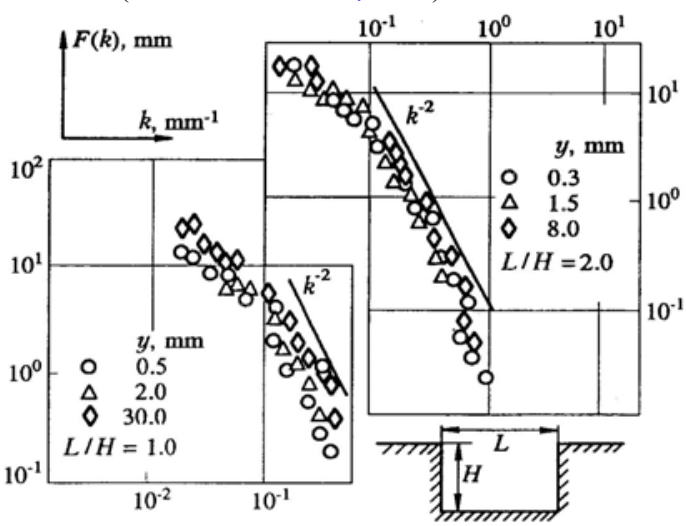

(a)

(b)

Fig. 2- Velocity fluctuation spectra in rectangular cavities

As is seen from this figure, in the range of wave numbers $k_{\mathrm{x}} \geq N^{-1}$ the "- 2 power law" is valid in accordance with our consideration in the near-wall zone $(y=0.5$ and $2.0 \mathrm{~mm}$ 
for the square cavity, $L / H=1$, and $y=0.3$ and $1.5 \mathrm{~mm}$ for the rectangular cavity with $L / H=2$ ).

Since there are no direct measurements of pressure fluctuations in flow we represent the available data by Simpson (1981) for pressure fluctuations on the wall in separating turbulent boundary layer Fig.3.

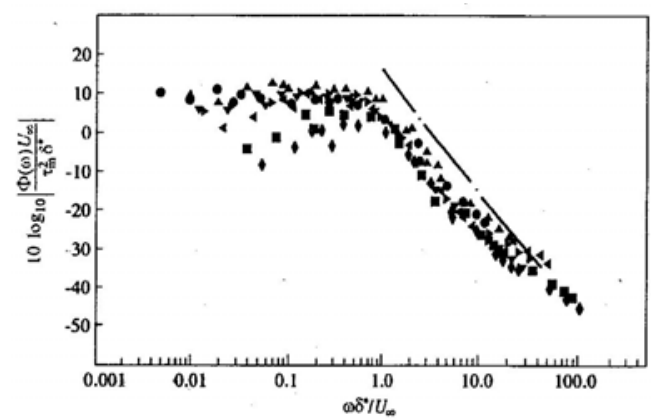

Fig.3- Wall pressure fluctuations in TSF

According to Simpson (1981), the phase velocity of pressure fluctuations was constant over this frequency range within the measurements accuracy. Therefore these data can be considered as supporting the "-3 power law" in the pressure fluctuations spectra. This law holds for spectra measured at the reattachment point of separated flow over a blunt flat plate (Kiya and Sasaki, 1987), Fig. 4.

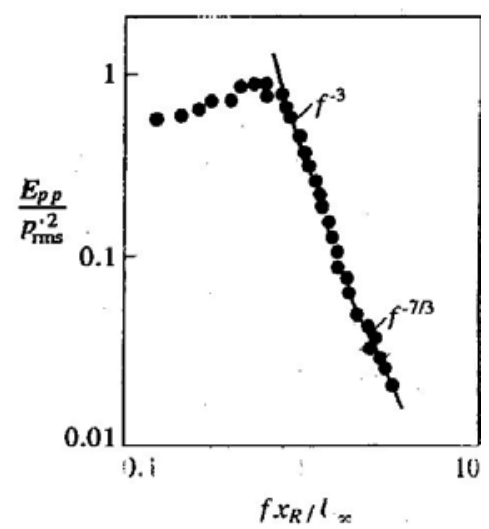

Fig. 4- Pressure fluctuations spectra at the reattachment point

Detailed measurements of the longitudinal velocity fluctuation intensities in the near-wall layer of recirculation flow behind a sudden increase in tube diameter are represented by Devenport and Sutton (1991). Figure 5 shows these data in coordinates $u_{*}=u^{\prime} / v_{*}^{\prime}$ and $y_{*}=v_{*}^{\prime} y / v\left(u^{\prime}\right.$ is the root-mean-square longitudinal velocity fluctuation, $v_{*}^{\prime}=\left(\tau_{*}^{\prime}\right)^{1 / 2}, \tau_{*}^{\prime}$ is the root-meansquare wall shear stress fluctuation, $x_{R}$ is the distance from the reattachment point). Near the wall the fluctuation velocity profiles merge into one curve $u_{*}=1.6 y_{*}^{1 / 2}$ in accordance with the similarity law (4). It should be noted that from dimensionality considerations for the "fluctuating friction velocity" $v_{*}^{\prime}$ it follows that $v_{*}^{\prime} \propto\left(v \alpha_{r m s}\right)^{1 / 3}$.

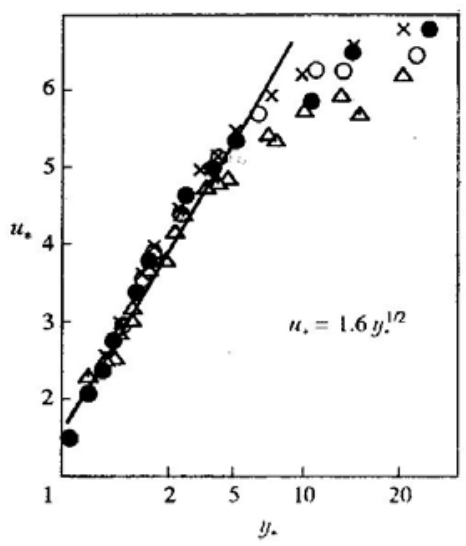

Fig. 5- Root-mean-square velocity fluctuation profile in the near-wall zone behind a sudden increase in tube diameter

In Fig. 6 the experimental velocity profiles in the nearwall layer of recirculation flow behind a step (Adams and Johnston, 1988) correspond well to the "half-power law" $U / U_{N}=\left(y / 3 \delta^{*}\right)^{1 / 2}$.

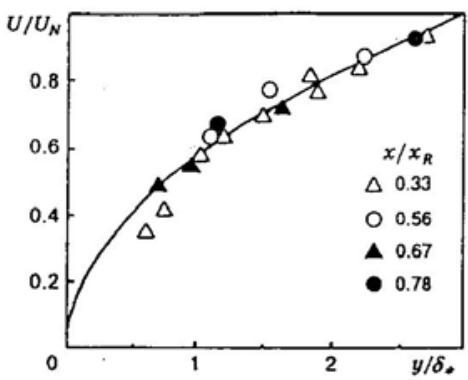

Fig. 6- Mean velocity profiles in the near-wall layer of recirculation flow behind a step

Fig. 7 shows the temperature profiles in the reattachment section of separated flow behind a rectangular obstacle on a flat surface (Pedishyus and Slanciauskas, 1987).

In this case $\quad \vartheta^{*}=\mathrm{St}^{-1 / 2}\left(T-T_{w}\right) /\left(T-T_{\infty}\right)$,

$y^{*}=\left(y U_{\infty} / v\right) \mathrm{St}^{1 / 2}, \quad \mathrm{St}=q /\left[\rho c_{0} U_{\infty}\left(T_{\infty}-T_{w}\right)\right]$,

$\delta^{*}$ is the displacement thickness of the incoming boundary layer, and $h$ is the obstacle height. 


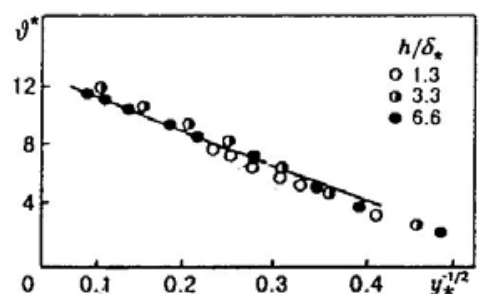

Fig. 7- Temperature profiles in the reattachment section behind a rectangular obstacle

To compare the heat transfer law (5) with experimental data the near-wall pressure gradient, $\alpha_{r m s}$ needs to be related to the parameters measured in experiments. It seems to be logical to relate $\alpha_{r m s}$ to the level of wall pressure fluctuations $p_{r m s}^{\prime}$ and the characteristic length scale of pressure disturbances $l_{p}, \alpha_{r m s}=p_{r m s}^{\prime} /\left(\rho l_{p}\right)$, so that the law (5), with $C_{p^{\prime}}=2 p_{r m s}^{\prime} /\left(\rho U^{2}\right)$ as the coefficient of wall pressure pulsations, becomes (Gorin and Sikovski, 1996)

$$
\mathrm{Nu}=\operatorname{const}\left(l_{p} / L\right)^{-1 / 3} C_{p^{\prime}}^{1 / 3} \operatorname{Re}^{2 / 3}
$$

The relationship $h_{w} \propto\left(p_{r m s}^{\prime}\right)^{1 / 3}$ between the wall heat transfer coefficient $h_{w}$ and the wall pressure fluctuations as it follows from Fig. 8 is in excellent
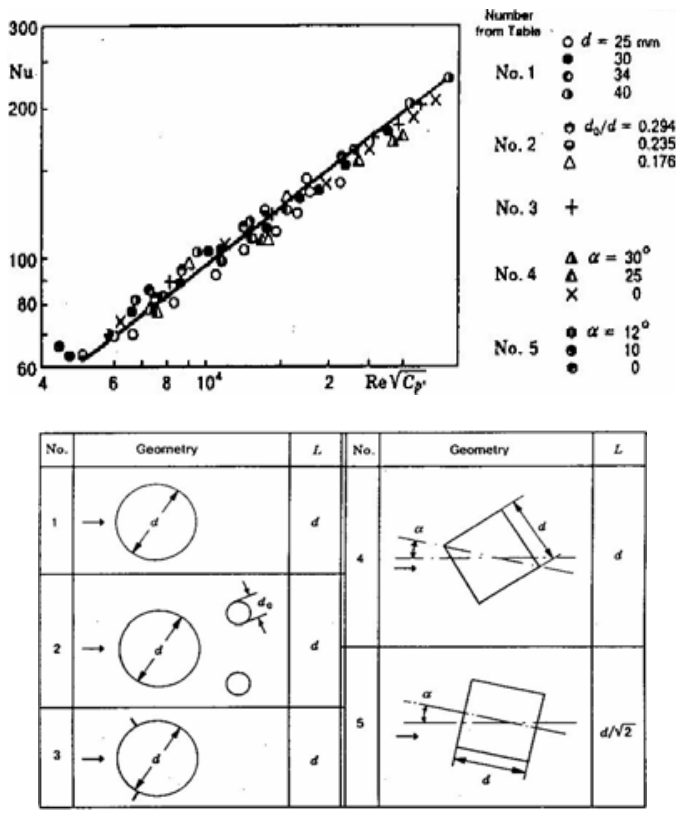

Fig. 8- Heat transfer from a cylinder and a square prism

agreement with experiments by Igarashi (1984) on heat transfer from a cylinder and a square prism and can be generalized by the curve
$\mathrm{Nu}=0.21 C_{p^{\prime}}^{1 / 3} \operatorname{Re}^{2 / 3}$

The law (5) makes it possible to generalize data by Ota and Kon (1974) on the local heat transfer on a blunt flat plate as well with the coefficient of proportionality only slightly different from that in Eq (6), Gorin and Sikovsky (1996, September):

$\mathrm{Nu}=0.23 C_{p^{\prime}}^{1 / 3} \operatorname{Re}^{2 / 3}$

A large body of experimental research on turbulent separated flows provides support for the similarity laws and the " $2 / 3$ power law" for heat and mass transfer (Gorin and Sikovsky, 2000; Gorin, 2005).

\section{CONCLUSION}

A general similarity theory developed for the near-wall zone of turbulent separated flow has demonstrated that the main characteristic features of transfer processes in this class of flow are well represented by the physical model based on an idea that flow in the near-wall zone is subjected to intense instantaneous accelerations induced by a large-scale vortex flow structure that prevents the formation of a sublayer with constant shear stress. The generated local large-scale wall pressure gradient equaled to the sum of mean pressure gradient due to the variation of the free-stream velocity and the large scale fluctuating pressure gradient induced by the large-scale vortices of separated shear layer plays the governing role in transfer mechanism formation. This approach provides an explanation to many experimentally observed facts. The similarity laws can serve as limiting laws for verification of validity turbulence models for separated flows: it was shown in Gorin and Sikovsky (1998) that standard $k-\varepsilon$ model has no solutions in the linear stress layer.

A similar dependence of heat and mass transfer upon Reynolds number has been observed in certain other turbulent flows - impinging jets, packed beds, tube banks in cross flow, compact heat exchangers-and this suggests the existence of classes of flows in which the transfer mechanisms are similar (Gorin and Sikovsky, 2000; Gorin, 2005).

\section{REFERENCES}

Adams, E. W. and J. P. Johnston (1988). Flow structure in the near-wall zone of a turbulent separated flow. American Institute of Aeronautics and Astronautics Journal, 26(8), 932-939.

Chang, P. (1970). Separation of Flow. Oxford, Pergamon Press.

Churchill, S. (2000). Progress in the thermal sciences: AIChE Institute Lecture. American Institute Chemical Engineers Journal, 46(9), 1704-1722. 
Devenport, W. J. and E. P. Sutton (1991). Near-wall behavior of separated and reattaching flows. American Institute of Aeronautics and Astronautics Journal, 29, 25-31.

Eaton, J. K. and J. P. Johnston (1981). A review of research on subsonic turbulent flow reattachment. American Institute of Aeronautics and Astronautics Journal, 19(9), 1093-1100.

Gorin, A. V. (2005). Heat transfer in turbulent separated flows. In T. Shakouchi, F. Durst, and K. Toyoda (Eds), Proceedings of International Conference on Jets, Wakes and Separated Flows, Toba-shi, JSME, No.05-201, pp. 445-450.

Gorin, A. V. and D. Ph. Sikovsky (1995). Similarity laws of transfer processes in turbulent separated flows, Russian Journal of Engineering Thermophysics, 5(2), 145-164.

Gorin, A.V. and D. F. Sikovski (1996). Turbulent heat-andmass-transfer model in the near-wall zone of separated flows, Journal of Applied Mechanics and Technical Physics, 37, pp. 370-380. [11]

Gorin, A.V. and D. Ph. Sikovsky (1996, September). Transfer processes in turbulent separated flows. In C.-J. Chen, C. Shin, J. Lienau, and R.J. Kung (Eds), Proceedings of the $6^{\text {th }}$ Int. Symposium on Flow Modeling and Turbulence Measurements, Tallahassee, Balkema/Rotterdam/Brookfield, pp. 881-888.

Gorin, A.V. and D. Ph. Sikovsky (1997). Scaling of wall turbulence in separated and reattached flows. In K. Hanjalic and T. W. J. Peeters (Eds), Proceedings of the $2^{\text {nd }}$ Int Symposium on Turbulence, Heat and Mass Transfer 2, Delft, 1997, pp. 121-128. Delft University Press.

Gorin, A. V. and D. Ph. Sikovsky (1998). On the ability of turbulence models to predict complex turbulent flows: application of scaling analysis. Proc of the $2^{\text {nd }}$ International EF Conference 'Turbulent Heat and Transfer 2', 2, pp. 63-71.

Gorin, A. V. and Sikovsky D. Ph. (2000). Fundamental laws of heat and mass transfer in turbulent flows with separated zones. Industrial Thermotechnics, 21(1), 10-17.

Gorin, A. V. and D. Ph. Sikovsky (2000, January) Heat and mass transfer correlations for turbulent separated flows. In M. S. Loknath et al (Eds). Proceedings of the Forth ISHMT-ASME Heat and Mass Transfer Conference and Fifteenth National Heat and Mass Transfer Conference 'Heat and Mass Transfer 2000', Puna, India, pp. 331337.
Hanjalic, K. (2005). A perspective on combining RANS and LES for computing complex flows and heat transfer. In T. Shakouchi, F. Durst, and K. Toyoda (Eds), Proceedings of International Conference on Jets, Wakes and Separated Flows, Toba-shi, JSME, 2005, No.05-201, pp. 25-34.

Igarashi, T. (1984). Correlation between heat transfer and fluctuating pressure in separating region of a circular cylinder, International Journal of Heat and Mass Transfer, 27, pp. 927-937.

Kiya, M. and Sasaki K. (1987). Structure of turbulent separation bubble. Journal of Fluid Mechanics, 177, 167-186.

Launder, B. E. (1988). On the computation of convective heat transfer in complex turbulent flows. Journal of Heat Transfer, 110, 1112-1128.

Ota, T. and N. Kon (1974). Heat transfer in a separated and reattached flow on a blunt flat plate. Journal of Heat Transfer, 94(4), 29-32.

Pedishyus, A. and A. Slanciauskas (1987). Near-wall Turbulent Heat Transfer. Vilnius: Mokslas.

Pletcher, R. H. (1988). Progress in turbulent forced convection, Journal of Heat Transfer, 110, 1129-1144.

Simpson, S. L. (1989). Turbulent boundary layer separation. Annual Review of Fluid Mechanics, 2, 205-234.

Simpson, R. L., Y. T. Chew, and B. G. Shivaprasad (1981). Structure of a separated turbulent boundary layer, Pt 1 \& 2. Journal of Fluid Mechanics, 113, 23-73.

Speciale, C. G. (1991). Analytical methods for the development of Reynolds-Stress closures in turbulence. Annual Review of Fluid Mechanics, 23, 107-157.

Varfolomeev, I. M., G. A. Glebov, and Yu. F. Gortyshov (1984). The structure of turbulent separated flow in rectangular cavity. In E. P. Volchkov (Ed.), Near-Wall Jet Flows, Institute of Thermophysics SB RAS, Novosibirsk, pp. 86-92. 